\title{
ELeGI \\ The European Learning Grid Infrastructure
}

\author{
Matteo Gaeta ${ }^{1}$, Pierluigi Ritrovato ${ }^{2}$, and Saverio Salerno ${ }^{3}$ \\ ${ }^{1,2}$ CRMPA, c/o Università di Salerno, Fisciano (SA), Italy \\ ritrovato@crmpa.unisa.it, gaeta@crmpa.unisa.it \\ ${ }^{3}$ Università di Salerno, Fisciano (SA), Italy, \\ salerno@unisa.it
}

\begin{abstract}
The purpose of this paper is to describe the ELeGI Project1. ELeGI has the ambitious goal of developing software technologies for effective human learning and promoting and supporting a learning paradigm shift. A new paradigm focused on knowledge construction using experiential based and collaborative learning approaches in a contextualised, personalised and ubiquitous way will replace the current information transfer paradigm focused on content and on the key authoritative figure of the teacher who provides information. We have chosen a synergic approach, sometimes called "human centred design", to replace the classical, applicative approach to learning. With consideration of humans at the centre, learning is clearly a social, constructive phenomenon. It occurs as a side effect of interactions, conversations and enhanced presence in dynamic Virtual Communities: experimental research concepts integrating new powerful developments of services in the Semantic GRID, the leading edge of currently available and future ICT technologies, with highly innovative and powerfully significant scenarios of human learning.
\end{abstract}

Keywords: Grid Architecture, GRID infrastructure, Complex Problem Solving and Grid applications, technology enhanced learning.

\section{PROJECT OBJECTIVES}

\section{Project Vision: Advancing Technology Enhanced Learning in Europe}

The overall aim of the project is to radically advance the effective use of technology-enhanced learning in Europe through the design, implementation and validation of a pedagogy-driven, service-oriented software architecture based on GRID technologies for supporting ubiquitous, collaborative, experiential-based, contextualised and personalised learning. Previous projects that have set out to improve learning through novel technologies have often failed to leave any significant mark because they did not give priority to the social, economic and technical perspectives of the key human actors. So, while the development and use of appropriate technology must be pedagogically driven, at the same time those involved in the formulation and evaluation of pedagogy must be made aware of, and shown by demonstration, state-of-the-art technological possibilities. We address this pervasive learning issue by explicitly listing the roles that actors play in the learning process and illustrate with reference to future learning scenarios. This provides us with a focus for formulating requirements in terms of didactical models, learning resources, services, quality of service, and usability for end-users. It also provides a clear reference for the technical context of the project - an open and flexible software architecture for creating learning environments that accommodate the roles implied by the new learning possibilities and that demonstrate state-of-the-art technologyenhanced learning.

\section{PEDAGOGICAL GOALS AND NEW LEARNING MODES}

In order to support the implementation of new learning modes related to ubiquitous, collaborative, experiential, contextualised and personalised learning, it is necessary to promote a paradigm shift in the general approach to teaching and learning.

\footnotetext{
${ }^{1}$ A 4 years Integrated Project submitted in the $1^{\text {st }}$ Call of the $6^{\text {th }}$ Framework Programme - Information Society Technology - of the European Commission. At the time of submission of this paper the project is in the Contract Negotiation phase and should start in January 2004.
} 
Currently, teaching and learning practices are based mainly on the information transfer paradigm. This focuses on content, and on the key authoritative figure of the teacher that provides information. Teachers' efforts are mainly devoted to find the best way for presenting content in order to transmit information to learners. This "product teaching oriented view" finds its perfect technical mirror in the "page oriented approach to the Web" where the goal is to produce more and "better" static pages for the consumption of interested students. Learning is then considered to be an activity which helps teachers to produce, and students to consume, multimedia books on the Web. This paradigm has been popular in earlier e-Learning projects, not because it is effective, but because it is easy to implement with basic Internet facilities and it does not require any change in the traditional roles of the actors.

The information transfer paradigm is well understood and well supported by existing e-Learning practice. In order to advance effective learning we will promote another paradigm that focuses on the learner and on new forms of learning. In our approach the learner has an active and central role in the learning process. Learning activities are aimed at facilitating the construction of knowledge and skills in the learner, instead of the memorisation of information. Information transfer will still obviously exist in the new paradigm, but only as a simple component, not the main goal. Accordingly we can say that the new paradigm subsumes the old one in its displacement.

Knowledge construction occurs through new forms of learning based on:

- the understanding of concepts through direct experience of their manifestation in realistic contexts (i.e. providing access to real world data) which are constructed from sophisticated software interfaces and devices, and represented as services;

- "social learning" - active collaboration with other students, teachers, tutors, experts or, in general, available human peers, by using different kinds of collaboration technologies, including enhanced presence.

In this approach collaboration is considered as a complex conversational process that goes far beyond a simple information exchange. In order to support such a "ubiquitous conversational process", one must consider the social context where the learning process occurs. Accordingly we do not consider the learner's ability in an abstract way, but relate it to a specific situation (the context). In this ambit the term "ubiquitous" does not refer simply to "anytime / anywhere", but more generally to the ability to support multiple diverse learning contexts and automatically adapt to them.

As we consider human learning as a social process, collaboration implies community membership, it means working together, providing added value, sharing and executing tasks in order to reach a common goal. Learning is no longer an isolated activity - it implies mutual trust, shared interests, common goals, commitments, obligations, exchanging of services, a genuinely proactive, motivated behaviour.

In order to foster these new approaches to learning we will create dynamic contexts where the learner "achieves" knowledge and skills in an active way instead of simply storing information. Communities will have the right to identify their goals, in terms of knowledge and skills to be acquired, instead of just asking an authority to define a curriculum for them. Goals will therefore genuinely correspond to needs, and be highly dependent on the local culture and its priorities.

According to this new learning paradigm we consider realism as the cornerstone of the learning environment. For example, highly realistic virtual scientific experiments have only recently become possible through use of advanced technology. Innovative aspects include the definition of a standard didactical model for the achievement and representation of such experiments. In this type of model a learner is immersed in a specific context, which through appropriate simulations, develops active learning processes with progressive abstraction levels, leading to the construction of their knowledge in a dynamic way. In this learning mode the student can also receive the support of the other users (collaborative aspects) and from the comparison with them, they can build a new "mediated" knowledge.

To complement this freedom in knowledge construction, we allow the definition of personalised and individualised learning paths. This means that in a specific context we need, from one side, to create learning conditions that are adequate for a learner's preferences (individualised learning) and, from the other, guarantee that the learner will reach a cognitive excellence through different learning paths according to their skills and knowledge. Accordingly, we will study and define specific models for representing knowledge that takes the learners preferred learning styles into account. A beneficial result of allowing learners the right to construct their own knowledge is that richer 
and more diversified learning contexts can arise, necessitating the dynamic integration of different kinds of information and communication technologies. The dynamics of intertwined, controlled and secure construction and use of subsequent versions of our systems, by skilled as well as unskilled human actors, and of the services enabling them, constitutes our methodological approach for successful adaptive technology-enhanced solutions.

\section{MEETING THE TECHNICAL CHALLENGE: THE GRID TECHNOLOGY}

In order to support ubiquitous, collaborative, experiential and contextualised learning in dynamic virtual communities a learning environment should provide the following features for learners:

- Collaboration; Socio-constructivist: group working should be routinely supported as well as the more traditional model of the solitary learner - this includes support for self-organising online communities who share common educational goals

- Experiential; Active Learning: learning resources should be interactive, engaging, and responsive - active learning and knowledge formation should be emphasised above simple information transfer

- Realism: real-world input should be easy to incorporate, as should simulations, ranging from simple interactive animations to immersive VR

- Personalised: students should find themselves at the centre of their online environment, with their individual needs addressed - the quality of the learning experience should be continually validated and evaluated

- Ubiquity and accessibility:

- wider, more flexible access to educational resources should be provided, often referred to as "anytime/anywhere" learning.

- multiple different types of devices, interfaces, and network connection types should be supported where possible

- Contextualised; Adaptive: appropriate learning contexts may be naturally be short-lived, as well as the more traditional static situations such as the classroom and the library - this calls for dynamicity in the creation of contexts

The pedagogical goals outlined above have highly demanding technical requirements, many of which are also the concerns of distributed systems research. Group working implies shared interactive resources, necessitating both concurrency control and awareness of others activities. Active learning requires interactive resources, many of which will only be engaging if they are suitably responsive - a quality of service (QoS) issue that depends on many components of a distributed system - the low-level infrastructure (hardware, OS, network), the middleware and the interface software. Concurrency control and interactive responsiveness can make conflicting demands on a system. Real world input, such as live stock market prices, or remote sensing data, makes a network connection mandatory, and this again raises QoS issues such as fault detection, masking and tolerance for the learning environment. Accessibility, as in anytime/anywhere, requires availability, which may be supported through replication of resources, but this creates further tensions with responsiveness and concurrency control due to the need to maintain state across replicas. Accessibility also means adapting to available capabilities. For example: can the same learning environment be delivered through low-bandwidth mobile devices and high-bandwidth multimedia workstations? Accessibility also means supporting special needs of the individual, such as disabilities. More generally, the individual user should be recognised and catered for, and this personalisation requires semantic tagging and profiling that can be difficult to formulate, both conceptually and in terms of machine representation. Standards efforts have been particularly slow in addressing this problem. Finally, contextualisation requires a move from the traditional view of an online learning environment as a stable long-lived entity (e.g. during the lifetime of a teaching module) - to one where the environment may evolve and change much more frequently, perhaps even several times a day - a dynamicity that is alien to current e-Learning products.

We believe that these technical requirements can best be addressed by building on the open distributed service model that has evolved as part of the Grid - why start from scratch if there is already a suitable and established base? The Grid a consolidation of selected distributed systems research output from the last twenty years.

The Grid was originally designed for e-Science and was primarily concerned with supercomputing applications, but the framework it engendered to realise effective sharing of distributed heterogeneous resources (OGSA: the Open Grid Services Architecture) is now being applied to many other areas, especially enterprise computing and eCommerce. Reciprocally, by progressing Grid technologies for learning, we will also contribute towards the 
advancement of the open Grid service model itself. We see the use of the Grid to support a paradigm shift in pedagogy to advance effective learning as a natural step in the recent historical progress of technology enhanced learning: Internet $->$ Web -> Grid.

OGSA leverages open standards including W3C, and provides an holistic view of Grid computing based on the concepts of 'Services', 'Distributed Collaboration' and 'Virtual Organisation'. At this point, new learning scenarios enter the picture: the user-centred, contextualised and experiential based approaches for ubiquitous learning imply the full exploitation of location-transparent access to distributed services such as simulation environments, realworld input, 3D visualisation systems and digital libraries, in the framework of a Virtual Organization. This allows a transition from current content-oriented e-Learning solutions towards a user-centred, collaborative model.

The next generation of Grid solutions will increasingly adopt the service-oriented model for exploiting commodity technologies. Its goal is to enable as well as facilitate the transformation of Information into Knowledge, by humans as well as - progressively - by software agents, providing the electronic underpinning for a global society in business, government, research, science, education and entertainment (semantic aspects) These efforts are sometimes referred to as the "Semantic Grid".

In summary, our proposal seeks to develop an OGSA compliant service oriented software architecture and realise a corresponding prototype infrastructure in order to support effective learning environments which exemplify the new paradigm.

\section{METHODOLOGY: TEST-BEDS AND DEMONSTRATORS}

Having described the overall objectives, the pedagogical goals and how we aim to meet the technical challenge, we now outline the methodology for the realisation and validation of ELeGI project. We have selected a particular set of demonstrators and test-beds representing scientific, social, economic and cultural cognate areas that include both formal and informal learning scenarios. The key difference between testbeds and demonstrators is that demonstrators already exist in non-Grid compliant forms, as relatively mature and well understood exemplars of the types of pedagogy ELeGI wishes to support, whereas testbeds are principally new departures, designed to test the ELeGI approach from conception to implementation and evaluation.

\section{Testbeds: Service Elicitation and Exploitation Scenarios}

As we are working towards a service-oriented architecture we refer to the test-beds as Service Elicitation and Exploitation Scenarios (SEES). The purpose of the SEES is to develop and gain insight into the processes involved from formulating pedagogic requirements to the implementing environments that meet these requirements. The following SEES, which are described in detail in the RTD section, are planned:

- Informal Learning
1) Alphabetisation for Durable Development
2) Learning and Training of Researchers in Organic Chemistry
3) e-Qualification by Open Universities

- Formal Learning

1) Master in ICT with remote teaching and tutoring activities (in collaboration with Carnegie Mellor University)

2) Physic course in the Open University

\section{Demonstrators}

Demonstrators differ from SEES in that they are based on the advanced prototypes which have adopted approaches congruent with the new paradigm, and where the pedagogical issues are already well understood. The purposes of the demonstrators are:

1) To provide evidence of the benefit coming from the adoption of didactical models based on socio-constructivist contextualised approach and to demonstrate the effectiveness of Grid technologies for implementing these didactical models.

2) to understand the engineering issues involved in implementing/porting existing solutions as OGSA-compliant software and services

3) to configure and customise these environments, for demonstrating effectiveness of specific research aspects

4) To prepare advanced contents for these environments

5) to provide working systems to elicit feedback and provide reference points within the project 
6) to act as "demonstrators" in support of publicity, dissemination and training activities

The demonstrators have been selected in order to maximise the benefits of the development work in that there is already a working non-Grid version. The three demonstrators planned are:

- Virtual Scientific Experiments for teaching high level mathematical courses.

- Learning Environment for Accountancy and Business Finance

- Learning Environment for Mechanical Engineering

\section{SUMMARY OF SCIENTIFIC AND TECHNICAL OBJECTIVES}

In summary, the project has three major goals.

Goal 1. To define new models of human learning enabling ubiquitous and collaborative learning, merging experiential, personalised and contextualised approaches.

Goal 2. To define and implement an advanced service-oriented Grid based software architecture for learning. This will allow us to access and integrate the different technologies, resources and contents that are needed in order to realise the new paradigm. This objective will be driven by the pedagogical needs and by the requirements provided by the test-beds (SEES) and informed by the experience gained through implementing the demonstrators.

Goal 3. To validate and evaluate the software architecture and the didactical approaches through the use of SEES and demonstrators. The project will build extensively on work already done, with the emphasis on creating new environments rather than components.

In order to reach these goals it will pursue the following objectives:

Goal 1

OB1. study, define and experiment with the new paradigm for formal and informal learning taking into account socio-cultural constructivist, personalised, contextualised, experiential and collaborative approaches

OB2. study, define, experiment and validate methodologies, techniques and standards for representing and managing knowledge construction in the learner during the learning process according to different learning styles (individualised and personalised learning);

OB3. study, define and validate methodologies for evaluating the effectiveness from both the pedagogical and usability point of view of these new learning approaches in different disciplines and contexts;

OB4. study, define and validate didactical learning models for virtual scientific experiments which take the learner's preferences and collaborative aspects into account;

\section{Goal 2}

OB5. design, develop and validate a GRID based software architecture for exploiting the new learning paradigm;

OB6. monitor and contribute to standards development for learning systems, pedagogical models, GRID technologies, semantic and knowledge technologies;

OB7. study and evaluate how the development of the semantic Web in general, and the semantic and knowledge GRID in particular, will impact on the project software architecture and new learning paradigm;

OB8. study, define and experiment with advanced conversational processes and enhanced presence technologies for collaborative learning in the new paradigm;

\section{Goal 3}

OB9. experiment and validate learning approaches, strategies, and technology infrastructure through different test-beds (SEES), using feedback for re-engineering and refining the context;

OB10. study and define strategies for the adoption of these new learning approaches and related technology facilitating the paradigm shift for the learning institutions;

OB11. demonstrate and validate how GRID technology facilitates the realisation of new learning paradigm and the implementation of Virtual Learning Communities;

OB12. demonstrate, customise and exploit new or re-engineered learning systems and solutions for citizens and organisations; 
OB13. facilitate the exploitation of European cultural and scientific resources though the Learning GRID infrastructure;

\section{PROJECT RESULTS AND THEIR EVALUATION}

The project results will be generated and evaluated through the use of the test-beds and demonstrators. The testbeds will evaluate the didactical approaches from cost/benefit and pedagogical points of view, and the support provided by the technology infrastructure in term of QoS, usability, scalability, interoperability and transparency of access to the distributed resources used for realising the didactical approaches. The Demonstrators will be used for stimulating the final users and the learning community in general about the potentialities offered by the realisation of a pedagogical driven service-oriented software architecture based on GRID technology (ELeGI)

In summary, the main ELeGl project results will be:

RS1. The service oriented GRID based Software Architecture;

RS2. Formalisation of didactical models for the new learning approaches;

RS3. Methodologies for evaluating the effectiveness of these new learning approaches from the pedagogical and usability points of view;

RS4. Methodologies and techniques for representing knowledge and for allowing personalised, individualised and collaborative learning;

RS5. Strategies for leading learning organisations and actors in the learning process to actualise the paradigm shift in learning approaches;

RS6. Prototypes for demonstrating the potentialities offered by the ELeGl technologies and methodologies;

RS7. New digital content in different contexts to support innovative learning approaches and in particular with respect to virtual scientific experiments;

RS8. Methodologies and techniques for making existing applications Grid-aware;

RS9. ELeGl evaluation and experimentation feedbacks;

RS10. Contribution to the technical standards in the Learning, semantic Web, and Grid domains;

RS11. Workshops, Conferences, Publications, Information Web sites, and Demonstrator Web sites for disseminating project results.

\section{INNOVATION RELATED ACTIVITIES}

According to the objectives sections the innovations that will be introduced with the ELeGl proposal can be classified in two different, but interrelated and objective- driven main groups. The first group is related to the pedagogical aspects the second group to the technology aspects.

The essence of innovations for pedagogical aspects is that in order to fully exploit e-learning processes we can not simply make the electronic transposition of traditional learning model. We need to investigate and define appropriate didactical models for e-learning. These models, based mainly on contextualised, experiential based and personalised approaches (socio-constructivist vision), will be defined taking into account in a synergic process both pedagogical and technological aspects fostering a learning paradigm based on socially situated, activitybased knowledge construction rather than information memorisation.

In particular, an innovative aspect is that our model will consider several characters of learning in a unitary approach. In the ELeGI proposal we will focus our attentions on the Active, Situated and Collaborative properties of learning.

- Active means that the learner is the principal actor of the process he/she takes the main decisions he/she learn choosing tools, exploring knowledge bases, accessing to virtual experiments, etc.

- Situated means contextualised with respect to activities motivated by goals, intentions, purposes, plans.

- Collaborative, means that the creation of the knowledge is a collaborative process evolving through interactions with colleagues, teachers, tutors, experts, instruments, etc.

In this frame standard models for experiential based learning will be proposed, in particular with respect to Virtual Scientific Experiments. 
Other innovative aspects are related to the possibility to personalise the learning process according to the learners' preferences and styles. In order to allow personalised learning processes we need to study and define methodologies for representing, through adequate knowledge structures (ontologies), and managing knowledge representing both the domain (the learning context) and the learner capabilities and skills itself including the representation of learner's attitudes, flaws as well as possible misunderstandings with respect to concepts and relations among several pieces of information related to a specific learning domain (e.g.: mathematics, physics, sociology, etc.). We are convinced we can now reach these objectives because we have already developed significant, even if quite limited, prototypes that present these features.

Exploiting these advanced knowledge representation structures, it will be possible to introduce innovative intelligent functionalities embedded into to the learning environment, namely the learning GRID infrastructure, realising a first concrete interpretation of the Ambient Intelligence vision in the learning domain.

Innovative will be the approach that we follow for implementing, experimenting and validating this new learning paradigm. Indeed, each and all the research activities will be aimed at satisfying user needs and will be validated through the execution of real size test-beds in an iterative approach, thus using the results as a feed-back as well as a feed-forward for improving and changing the models defined.

It is clear that in order to reach these ambitious objectives we need to design and implement a very powerful technological infrastructure. The widest is the potential learning audience, the most sophisticated should be the technology and, at the same time, the simplest to use. From this point of view the main innovation is the use of GRID technologies for implementing a service oriented software infrastructure. The use of GRID technologies implies the adoption of a framework based approach for designing our infrastructure. This means to raise the level of abstraction in the infrastructure design:

- trying to be as much as possible neutral respect to technological evolutions,

- focusing our attention on the definition of interfaces and behaviours of "learning services" in a standard way and

- implementing an infrastructure that will incrementally support the realisation and actualisation of our new learning paradigm.

We are well aware, as we said in previous sections, that for actualising this new learning paradigm we need to integrate and coherently orchestrate several kinds of technologies and existing software solutions in an innovative way.

Investigating and experimenting with the use of GRIDs as technology glue for implementing dynamic service oriented Virtual Learning Organisations (VLO) also introduces potential innovations fed to the technological level: the service elicitation scenarios will continuously feed the technologists with requirements for new services described at the abstraction level of the pedagogy, to be translated into combinations of services at the abstraction level of technologies. The continuous "translation" process from the one to the other abstraction level is exactly the core of the synergies declared as the innovative approach of ELeGI.

Finally, innovations for the GRID technologies strictly connected to support dynamic Virtual Learning Organisations emerge from the study and analysis of semantic, trust \& security aspects. In order to implement dynamic VLOs, we need to define mechanism for services discovery as well as to automatically for "understanding" the capabilities of these discovered services. In order to do that, we need to define standards for describing GRID serviced semantically. This is one of the hottest research topics in the GRID domain and we believe that with the ELeGI proposal we can contribute substantially to the progress in this field. Moreover, the exploitation of this semantic enrichment of services will be fundamental for supporting collaboration and interactivity as well as for creating the necessary awareness for implementing a Learning Ambient Intelligent vision.

It is clear that VLOs, due to their dynamic nature, needs policy driven, configurable and powerful security mechanism. Moreover, without security this kind of infrastructure will be confined to the research community and will never be used in the business domain. Grid technologies, at the data level, are mature enough but their introduction is not yet diffused as it may be since work has to be done in order to make GRID technologies usable from a secure point of view. 
Conversational processes, enhanced presence and the Semantic Grid are all activities identified in ELeGI and assigned to highly qualified partners, with an outstanding record of excellence in research, both fundamental and applied. The three aspects run together: learning services, the ones needed for enabling effective learning to occur, have to be first identified by means of conversational processes by humans in virtual communities accompanied by enhanced presence in order to keep motivation and commitment to the community's goals high and performing. Identified services have then to be progressively transformed into software, i.e. the semantics of services has to be constructed in order to make an infrastructure that supports those services. It will be vital for the project's success that the life cycle of service identification, design, implementation and exploitation will be understood by each actor in the loop. Looking at the forest of available technologies, anyone is discouraged by the impressive number and complexity of each component, language and device.

\section{THE OVERALL APPROACH}

The picture below shows the organisation of the RTD activities and the relations existing among the research activities.

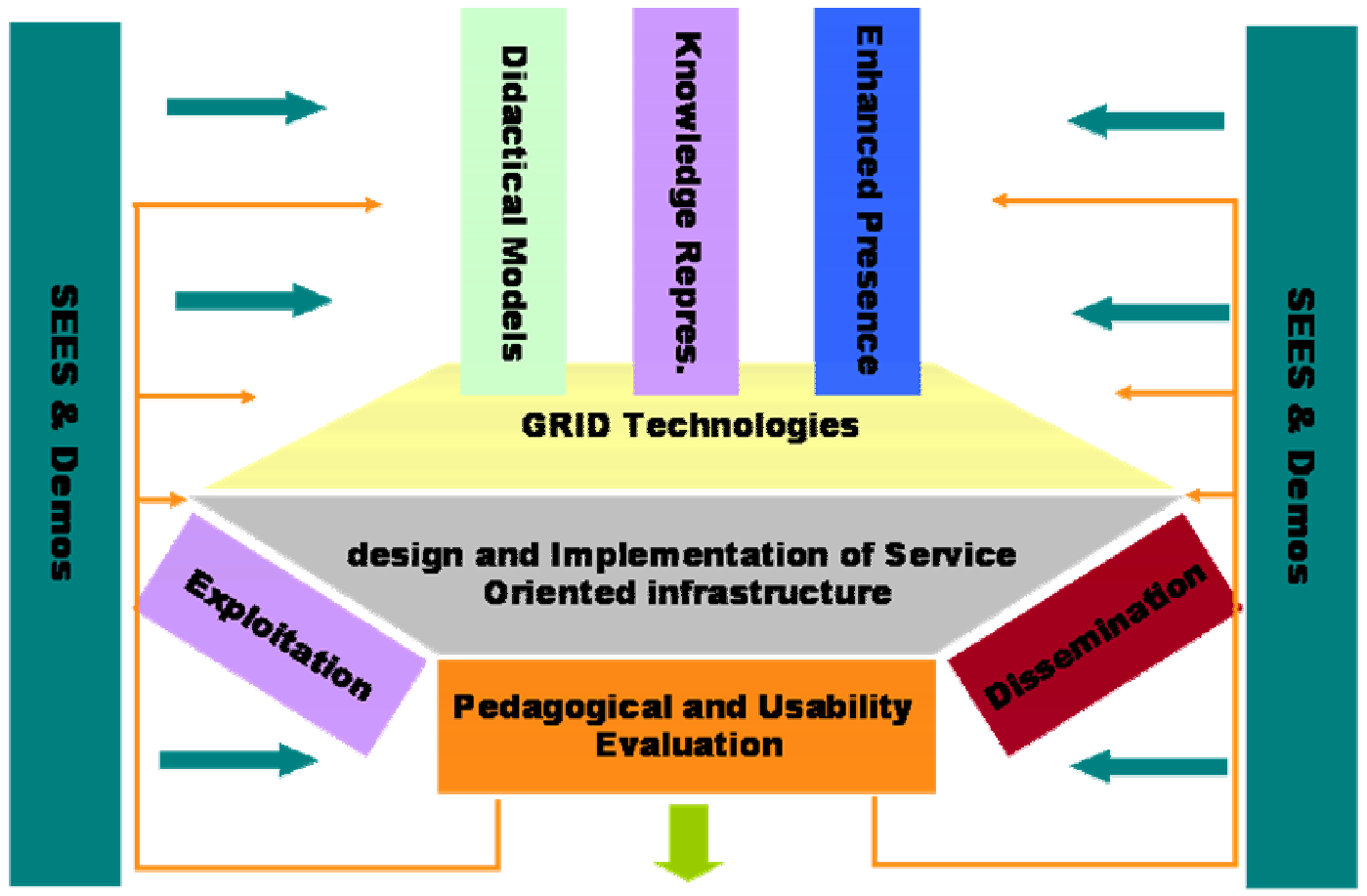

Learning GRID Infrastructure 


\section{Participants List}

\begin{tabular}{|c|l|c|c|}
\hline$\#$. & \multicolumn{1}{|c|}{ Name } & Short Name & Country \\
\hline 1 & SchlumbergerSEMA (Financial Coordinator) & SLBSema & Spain \\
\hline 2 & $\begin{array}{l}\text { Centro di Ricerca in Matematica Pura ed Applicata (Scientific } \\
\text { Coordinator) }\end{array}$ & CRMPA & Italy \\
\hline 3 & $\begin{array}{l}\text { Centro di Eccellenza su Metodi e Sistemi per l'Apprendimento } \\
\text { e la Conoscenza }\end{array}$ & CE & Italy \\
\hline 4 & Université Montpellier 2 & UM2 & France \\
\hline 5 & Telindus Group NV & THTI & Belgium \\
\hline 6 & University of Southampton & UOS & UK \\
\hline 7 & University of St. Andrews & USTAN & UK \\
\hline 8 & Karl-Franzens-Universitaet Graz im Rahmen der & KFU & Austria \\
\hline 9 & Teilrechtsfaehigkeit gemaess & & \\
\hline 10 & The Open University & OU & UK \\
\hline 11 & Universität Stuttgart & CCLRC & UK \\
\hline 12 & Ruhr Universität Bochum & USTUTT & Germany \\
\hline 13 & Kaunas Technology University & RUB & Germany \\
\hline 14 & Communication \& Systèmes - Systèmes d'information & KTU & Lithuania \\
\hline 15 & Friedrich-Alexander-Universität Erlangen-Nürnberg,FIM & CS-SI & France \\
\hline 16 & NeuesLernen & FAU & Germany \\
\hline 17 & Unilenic Open University & HOU & Greece \\
\hline 18 & Research and Education Society in Information Technologies & UNIVDUN & UK \\
\hline 19 & Facultés N.D. de la Paix & AIT & Greece \\
\hline 20 & Centre National de la Recherche Scientifique & CNRS & Belgium \\
\hline 21 & Université De Pau et des Pays De L'adour-lut de Bayonne & UPPA & France \\
\hline 22 & Ecole Centrale de Paris & ECP & France \\
\hline 23 & European Microsoft Innovation Centre & EMIC & Germany \\
\hline
\end{tabular}

